\title{
ANÁLISIS DE VARIABLES ANTROPOMÉTRICAS Y BIOMECÁNICAS QUE INFLUYEN EN LA VELOCIDAD, DE NIÑOS QUE PRACTICAN FUTBOL ENTRE 10 Y 14 AÑOS DEL CLUB SANTIAGO WANDERERS DE VALPARAÍSO \\ ANALYSIS OF ANTHROPOMETRIC VARIABLES AND BIOMECHANICAL INFLUENCE THE RATE OF CHILDREN WHO PLAY FOOTBALL BETWEEN 10 AND 14 YEAR OF SANTIAGO WANDERERS CLUB OF VALPARAÍSO
}

Barraza Gómez, Fernando1; Cajas Luna, Boris2; Inostroza Bailles, Alex1; López Montes, Branco1 \& Rodríguez Moraga, Diego1.

1 Laboratorio Motricidad Humana, Escuela de Educación Física, Pontificia Universidad Católica de Valparaíso.

2 Escuela de Educación Física, Universidad de Viña del Mar.

BARRAZA G. F.; CAJAS L. B.; INOSTROZA B. A.; LOPEZ M. B. \& RODRIGUEZ M. D. análisis de variables antropométricas y biomecánicas que influyen en la velocidad, de niños que practican futbol entre 10 y 14 años del club Santiago Wanderers de Valparaíso.

Mot. Hum., 12(2): 32-36, 2011.

\section{RESUMEN}

Se presentan en el estudio los resultados del análisis de variables antropométricas (talla, peso y longitud del miembro inferior) y biomecánicos (frecuencia de pasos y amplitud de zancada), en el cual se pretendió encontrar la relación de estas variables con la velocidad y entre ellas. El estudio se desarrolló con una muestra de 75 sujetos varones que practican fútbol al menos 3 veces por semana, pertenecientes a las divisiones menores del club Santiago Wanderers de Valparaíso. A su vez, los sujetos del estudio se dividieron por edad en 4 categorías: (que son las que participan en el Campeonato Nacional de Fútbol Joven) Sub 14 (17 Sujetos), Sub 13 (17 Sujetos), Sub 12 (18 Sujetos), Sub 11 (23 Sujetos).En el caso de las categorías más grandes (Sub 14 y Sub 13), aquellas variables biomecánicas dieron como resultado la mayor explicación de la velocidad y en las otras categorías (Sub 12 y Sub 11) no se encontraron variables explicativas a la velocidad.

Palabras clave: Futbol, Velocidad, Biomecánica, Antropometría.

\section{INTRODUCCIÓN}

El fútbol es un deporte colectivo, de oposición y que se juega casi exclusivamente con los pies. Cuenta con una gran cantidad de adeptos a nivel mundial, lo que lo transforma en un deporte muy popular, haciendo incluso que muchos lo denominen como el deporte más popular del mundo. La evolución de éste, ha hecho que cada vez sea más necesario realizar esquemas de juego que no sólo aseguren el resultado de un partido o campeonato, sino que además intenten exhibir una forma de juego vistosa y rápida. Es por ello que hoy en día el fútbol se caracteriza por ser jugado a un ritmo intenso, y la velocidad se ha transformado en uno de los aspectos fundamentales en su desarrollo, ya sea para obtener ventaja sobre el rival o mostrar un juego vistoso que agrade a la afición. Eso conlleva a que el jugador de fútbol debe encontrar su mejor desempeño a una gran velocidad. Ya no es inusual ver que un defensa lateral llegue al área contraria en una jugada de ataque o que el delantero al perder un balón retroceda rápidamente en busca de su recuperación. Estas situaciones se muestran dependientes de la velocidad y a la vez, ésta depende de diferentes variables que Cianciabella (1) identifica como la coordinación neuromuscular, las proporciones morfológicas (estructura ósea, peso), velocidad de contracción de los músculos, cantidad de fibras rápidas, técnica de carrera, elasticidad muscular, el conocimiento técnico- táctico y el grado de atención. Existen también factores externos como temperatura, terreno, altura, etc.

Entendiendo la importancia de la velocidad en el fútbol actual, Aon (2) señala que la velocidad es una de las cualidades físicas más importantes para el fútbol, pero que debería estar pura y exclusivamente al servicio de la inteligencia futbolística y no del correr por correr. Así, en este estudio buscamos identificar cuáles de las variables influyen de manera más significativa respecto a la velocidad de un jugador de futbol. Respecto a las variables influyentes en la velocidad, Ruis (3) señala que hay una serie de componentes como: antropometría del sujeto, tipo y distribución de fibras musculares y estimulación 
BARRAZA G. F.; CAJAS L. B.; INOSTROZA B. A.; LOPEZ M. B. \& RODRIGUEZ M. D. análisis de variables antropométricas y biomecánicas que influyen en la velocidad, de niños que practican futbol entre 10 y 14 años del club Santiago Wanderers de Valparaíso. Mot. Hum., 12(2): 32-36, 2011.

nerviosa del músculo. En este estudio buscaremos determinar la relación existente entre variables antropométricas como Talla, Longitud del miembro inferior, Peso y otras de carácter biomecánico tales como Amplitud y Frecuencia de pasos, así como también poder definir cual o cuales de éstas podrían tener una mayor influencia en la velocidad, vista como variable dependiente de la investigación.

Respecto a la edad de los sujetos dentro de esta investigación, Weineck (4) en el ámbito infantil y juvenil especialmente en las edades comprendidas entre los 8 y los 16 años - la plasticidad de la corteza cerebral y la inestabilidad morfológica del sistema nervioso permiten una muy buena formación básica en lo que se refiere a las capacidades de velocidad. A esto Grosser (5) añade que entre los 8-10 y 12 años, se pasa por una fase de fuerte desarrollo, de manera que los niños en estas edades podrán concentrarse más y más tiempo, dado que su voluntad y motivación por aprender y mejorar es mayor. La velocidad es una variable multifactorial en la cual participan una serie componentes que Ruis (6) descompone en: antropometría del sujeto, tipo y distribución de fibras musculares y estimulación nerviosa del músculo, además Ferro (7) añade dos componentes más los cuales son Longitud de zancada y Amplitud de zancada, componentes biomecánicos que en este artículos serán foco de estudio, teniendo en cuenta que una buena técnica tiene una importancia bastante relevante como señala Cissik (8) "Una buena técnica permitirá que un corredor mueva sus extremidades rápida y seguramente. Una técnica deficiente resultará en una pobre eficacia de movimiento, producirá fuerzas de frenado, y la sobrecarga de ciertos músculos y articulaciones lo que posiblemente derivará en una lesión."

\section{MATERIAL Y METODOS}

Fueron considerados un total de 75 sujetos varones (edad 10 a 14 años) en las categorías Sub 14 (17 Sujetos), Sub 13 (17 Sujetos), Sub 12 (18 Sujetos), Sub 11 (23 Sujetos). Los sujetos fueron evaluados antropométricamente, el primer dato obtenido fue la estatura, se utilizó un estadiometro portátil validado por el Laboratorio de Motricidad Humana de la PUCV, controlando la altura y posicionado verticalmente. Para la medida del peso corporal, se utilizó una balanza digital, la siguiente medida corresponde a la longitud del miembro inferior para ello se procede a marcar el punto trocantéreo que corresponde al punto más superior del trocánter mayor del fémur, luego de esta operación lo que se hace es utilizar un calibre con bandas deslizantes, Campbell 20 (antropómetro largo), el cual se ubica un extremo en la zona identificada anteriormente del trocánter y el otro extremo se extiende hasta la planta del pié del sujeto parado. El segundo paso es evaluar las velocidades de los sujetos con fotocélulas (Marca MEYDEP, Modelo MAC 1207) se procedió primeramente el grupo evaluador a disponer 2 fotocélulas al inicio del recorrido y 2 fotocélulas a una distancia final de 20 metros. Estas fotocélulas se ubican a los costados del trayecto a recorrer, con una distancia aproximada de 4 metros entre sí. A su vez se filma el recorrido del evaluado con 2 cámaras grabadoras ubicadas una a los 10 metros a un costado del recorrido para observar el plano lateral del sprint y una cámara ubicada al final del recorrido que observa el sprint con un plano frontal. Los sujetos son previamente registrados en el software del programa indicando su nombre. En cuanto a la recolección de los datos a utilizar el software del programa entrega los datos de tiempo empleado y promedio de velocidad de cada sujeto registrado. Mientras que los datos de amplitud y frecuencia de pasos son obtenidos luego de contar la cantidad de contactos que realiza cada sujeto utilizando las grabaciones, las cuales son visualizadas detenidamente con el programa Kinovea. El tiempo empleado para recorrer los 20 metros es dividido por la cantidad de contactos para obtener la frecuencia, mientras que la amplitud es obtenida luego de dividir la distancia de la carrera (20 metros) por la cantidad de contactos.

\section{RESULTADOS}

Para el análisis de los resultados obtenidos, se utilizó un análisis descriptivo, de correlación y regresión.

A partir del análisis descriptivo se puede apreciar que la variabilidad de los datos, representada por la varianza, es mayor en las variables antropométricas (peso, talla, longitud miembro inferior) que en las variables biomecánica (frecuencia de pasos y amplitud de zancada) (Tabla I). 
BARRAZA G. F.; CAJAS L. B.; INOSTROZA B. A.; LOPEZ M. B. \& RODRIGUEZ M. D. análisis de variables antropométricas y biomecánicas que influyen en la velocidad, de niños que practican futbol entre 10 y 14 años del club Santiago Wanderers de Valparaíso. Mot. Hum., 12(2): 32-36, 2011.

\begin{tabular}{|lrrrr|r|}
\hline Variable & Mín & Máx & Med & Var & N \\
\hline Frecuencia de Pasos (Hz) & 3,81 & 4,98 & 4,41 & 0,07 & 75 \\
Amplitud de zancada (mts) & 1,18 & 1,54 & 1,29 & 0,01 & 75 \\
Talla (cm) & 133,60 & 176,00 & 152,57 & 106,06 & 75 \\
Peso (kg) & 32,00 & 75,00 & 46,19 & 100,84 & 75 \\
Long. Miembro Inf. (cm) & 70,00 & 94,00 & 80,82 & 28,20 & 75 \\
Velocidad (mts/seg) & 5,03 & 6,57 & 5,64 & & 75 \\
\hline & & & \\
\hline
\end{tabular}

Además se evidencia un comportamiento ascendente en el valor de las variables antropométricas con respecto a la edad, lo cual no ocurre de igual manera con las variables biomecánicas ya que estas presentan un comportamiento irregular con respecto al aumento de la edad (Tabla II). Del análisis de correlación se puede apreciar que las correlaciones más altas obtenidas corresponden a las existente entre longitud de miembro inferior - talla (.921) y peso - longitud de miembro inferior $(0,789)$. (Tabla III).

\begin{tabular}{|c|c|c|c|c|}
\hline Variables & Sub 14 & Sub 13 & Sub 12 & Sub 11 \\
\hline Velocidad (mts/seg) & 6,05 & 5,58 & 5,64 & 5,39 \\
\hline Frecuencia (HZ) & 4,46 & 4,32 & 4,52 & 4,34 \\
\hline Amplitud (mts) & 1,36 & 1,3 & 1,25 & 1,25 \\
\hline Peso $(\mathrm{Kg})$ & 55,8 & 50,0 & 42,8 & 38,9 \\
\hline Talla (cm) & 162,8 & 159,1 & 149,4 & 136,6 \\
\hline M. Inferior (cm) & 85,5 & 84,0 & 78,8 & 76,5 \\
\hline
\end{tabular}

La variable frecuencia de pasos presenta relaciones inversas con las variables de talla $(-0,188)$ y longitud del miembro inferior $(-0,336)$. Por el contrario, la variable amplitud de zancada presenta relaciones directas, pero no altamente significativas con estas mismas variables $(0,671)$ y $(0,680)$ respectivamente.

\begin{tabular}{|c|c|c|c|c|c|c|}
\hline & $\begin{array}{r}\text { Frecuencia } \\
\text { de Pasos }\end{array}$ & $\begin{array}{r}\text { Amplitud } \\
\text { de Zancada }\end{array}$ & Talla & Peso & $\begin{array}{r}\text { Longitud Miembro } \\
\text { Inferior }\end{array}$ & Velocidad \\
\hline Frecuencia de Pasos & 1 & -0.571 & -0.188 & -0.108 & -0.336 & 0.454 \\
\hline Amplitud Zancada & -0.571 & 1 & 0.671 & 0.553 & 0.680 & 0.469 \\
\hline Talla & -0.188 & 0.671 & 1 & 0.893 & 0.921 & 0.531 \\
\hline Peso & -0.108 & 0.553 & 0.893 & 1 & 0.789 & 0.485 \\
\hline Longitud Miembro & & & & & & \\
\hline Inferior & -0.336 & 0.680 & 0.921 & 0.789 & 1 & 0.382 \\
\hline Velocidad & 0.454 & 0.469 & 0.531 & 0.485 & 0.382 & 1 \\
\hline
\end{tabular}


BARRAZA G. F.; CAJAS L. B.; INOSTROZA B. A.; LOPEZ M. B. \& RODRIGUEZ M. D. análisis de variables antropométricas y biomecánicas que influyen en la velocidad, de niños que practican futbol entre 10 y 14 años del club Santiago Wanderers de Valparaíso. Mot. Hum., 12(2): 32-36, 2011.

Con respecto a la relación entre las variables estudiadas y la velocidad, se puede apreciar que esta tiene una relación directa con todas ellas, sin embargo dichas relaciones no son altamente significativas. En este sentido, las relaciones que más se destacan son entre la velocidad - talla (.531), velocidad - amplitud de zancada (.469) y velocidad - frecuencia de pasos (.454). (Tabla III).

El análisis de regresión se utilizó para poder determinar cuáles son los factores que inciden significativamente en la velocidad de la carrera de Sprint en 20 metros. El método ocupado para este fin correspondió al modelo hacia atrás en el análisis global, mientras que para el análisis por categoría se utilizó el modelo paso a paso.

En este sentido, el modelo ajustado resultante a nivel global fue el siguiente:

\section{Velocidad $=-5,869+1,316 \times$ FC $+4,439 \times$ Ampl. Zanc $+0,015$}

De los resultados del análisis de regresión a nivel global, el modelo completo mencionado corresponde a todas las variables bajo estudio, las cuales explican en un 99,6\% la velocidad. El resto de los modelos corresponde al modelo anterior menos una variable, por lo tanto,

En lo que respecta al análisis de regresión por categoría con el modelo paso a paso, en el cual en vez de restar, se suman variables, las categorías sub 14 (tabla V) y sub 13 (Tabla VI) demuestran que son las variables de frecuencia de pasos y amplitud de zancada en su conjunto son las que explican de mejor manera la velocidad $(99,1 \%)$ y $(99,8 \%)$ respectivamente.

En el caso de las categorías sub 12 y sub 11, ninguna variable resultó explicativa de la velocidad.

Tabla V: Variaciones del R2, asociadas a las variables introducidas

Tabla VI: Variaciones del R2, asociadas a las variables introducidas

\section{DISCUSIÓN}

El aumento de los valores en los parámetros de las variables antropométricas de talla, peso y longitud del miembro inferior encuentra respuesta en que a medida que aumentamos de edad, bajo índices normales de crecimiento, se aumenta la talla y el nivel de desarrollo, lo cual afecta a las otras dos variables generando un aumento de estas más aun en estas edades de máxima velocidad de crecimiento como señala Requejo, A. y Ortega, R. (9) que es a los 13.5 años (edad media) y Marin, Z. (10) señala la edad media de 14 años, teniendo claro que cada individuo tiene un ritmo de crecimiento diferente al otro, es decir, no todos crecen al mismo tiempo y al mismo nivel.

Con respecto a las variables de frecuencia de pasos y amplitud de zancada el comportamiento de sus resultados tienen un desarrollo de dependencia multifactorial.

Dentro de las variables estudiadas, aquellas que tienen mayor relación con la velocidad son, la variable de amplitud de zancada, la cual sumada a la variable de frecuencia de pasos explican de mejor manera la velocidad alcanzada en una carrera de $20 \mathrm{mts}$. Con respecto a la variable de longitud del miembro inferior planteada en la investigación, en relación con la velocidad, podemos señalar que la longitud miembro inferior no presenta una relación lineal significativa con la velocidad, pero sí con la amplitud de zancada, la cual se relaciona directamente con la velocidad, es por ello que creemos que la longitud del miembro inferior afecta de una u otra manera a la variable velocidad. Siguiendo con la importancia de las variables de frecuencia de pasos y amplitud de zancada, es que debemos concluir que entre ellas no existe una predominancia de una sobre la otra en relación a la velocidad, sino que estas deben interactuar de manera conjunta y proporcionada, según cada sujeto, para poder tener un buen desarrollo de la velocidad.

De acuerdo al análisis de correlaciones obtenidas en este estudio podemos señalar, que para entender el comportamiento de las variables de frecuencia de pasos y amplitud de zancada debemos tener en cuenta los factores antropométricos del sujeto de estudio, nos referimos a talla, peso y longitud del miembro inferior, las cuales presentan directa relación con la amplitud de zancada, e inversa relación con la frecuencia de pasos, por lo que aquellos sujetos de menor talla presentan una mayor frecuencia de pasos, lo que conllevaría a tener que mejorar la amplitud de zancada para lograr así el equilibrio entre las variables mencionadas. Lo mismo, a la inversa, pasaría en el caso de los sujetos con mayor talla, los cuales al presentar una mayor amplitud de zancada deberán enfocarse en mejorar la frecuencia de pasos. 
BARRAZA G. F.; CAJAS L. B.; INOSTROZA B. A.; LOPEZ M. B. \& RODRIGUEZ M. D. análisis de variables antropométricas y biomecánicas que influyen en la velocidad, de niños que practican futbol entre 10 y 14 años del club Santiago Wanderers de Valparaíso. Mot. Hum., 12(2): 32-36, 2011.

Finalmente indicamos que las variables de amplitud de zancada y frecuencia de pasos son aquellas que tienen mayor incidencia en la velocidad, pero estas a su vez son dependientes de la antropometría del sujeto concordando con lo expresado por Romagnoli, Sánchez, Díaz, Arduini
(11), los cuales concluyen que para la consecución de la máxima velocidad, es necesario encontrar para cada atleta la frecuencia y la longitud de pasos óptimas en función de sus características antropométricas.

\title{
REFERENCIAS BIBLIOGRAFICAS
}

1. Cianciabella, J.E. (1997). La velocidad en el futbol. Criterios para el desarrollo de la velocidad mental, Educación Física y Deportes, N4.

2. Aon, Justo. La velocidad en el futbol. criterios para el desarrollo de la velocidad, http://www.fuerzaypotencia.com.

3. Ruis, J. (2005). Metodología y técnicas de atletismo. Barcelona: Paidotribo.

4. Weineck. J. (1994). Futbol total. El entrenamiento físico del futbolista Vol II. Barcelona.

5. Grosser, M. (1992) Entrenamiento de la velocidad., Barcelona: Ed. Martínez Roca.

6. Ruis, J. (2005). Metodología y técnicas de atletismo. Barcelona: Paidotribo.

7. Ferro, A. (2001). La carrera de velocidad: metodología de análisis biomecánico. Madrid: Esteban sanz, S.L.

8. Cissik, John. (2004). Desarrollo de la Técnica y la Velocidad para la Carrera.

9. Requejo, A. y Ortega, R. 2002, Nutrición en la adolescencia y juventud, Madrid: Complutense

10. Marin, Z., 2008, Elementos de nutrición humana.,Costa Rica: EUNED

11. Romagnoli, M. Sánchez, J. Díaz, V. Arduini, A. (s/f). Análisis de la amplitud y frecuencia de paso de carrera a diferentes velocidades en deportistas amateurs.

\begin{abstract}
In the study are presented the results of the analysis of anthropometric variables (height, weight and leg length) and biomechanical (Cadence and stride length), which was intended to find the relationship of these variables with the speed and between them. The study was conducted with a sample of 75 male subjects who play football at least 3 times a week, belonging to the lower divisions of the club Santiago Wanderers of Valparaiso. In turn, the study subjects were divided by age into 4 categories: (which are participating in the National Football League Young) Sub 14 (17 subjects), Under 13 (17 subjects), Under 12 (18 subjects), Sub 11 (23 subjects). in the case of the larger categories (Under 14 and Under 13), those biomechanical variables resulted in further explanation of speed and in the other categories (Sub 12 and Sub 11) no explanatory variables found to speed
\end{abstract}

Key words: Football, Speed, anthropometric, biomechanical

\section{Dirigir Correspondencia a:}

Fernando Barraza Gómez

Email: fernando.barraza@ucv.cl

Fono: 032-2274381

Avda. El Bosque 1290, Santa Inés, Viña del Mar.

RECIBIDO: 26-11-2011

ACEPTADO: 1-12-2011 